\title{
1 Gbaud QPSK Wireless Receiver using an Opto- Electronic Mixer
}

\author{
Ahmad W. Mohammad, Haymen Shams, Chin-Pang Liu, Katarzyna Balakier, Chris Graham, Michele Natrella, \\ Alwyn J. Seeds, and Cyril C. Renaud \\ Department of Electronic and Electrical Engineering \\ University College London (UCL) \\ London, UK \\ ahmad.mohammad@ucl.ac.uk
}

\begin{abstract}
This paper presents the first demonstration of an uni-travelling carrier photodiode (UTC-PD) used as a receiver of a wirelessly transmitted quadrature phase shift keying (QPSK) signal. In this demonstration, a 1 Gbaud QPSK signal centered at 33.5 $\mathrm{GHz}$ was transmitted over a wireless distance of $1.4 \mathrm{~m}$. At the receiver, an UTC-PD is used to down-convert the RF signal to an intermediate frequency (IF) of $9.5 \mathrm{GHz}$ by mixing the RF signal with a heterodyne signal at $24 \mathrm{GHz}$. The down-converted signal is captured by the real time scope for further digital signal processing. The error vector magnitude (EVM) of the demodulated signal was measured to be $18 \%$, which corresponds to a bit error rate (BER) of $10^{-8}$.
\end{abstract}

Keywords - uni-travelling carrier photodiode; wireless receiver; optically pumped mixing; $5 G$.

\section{INTRODUCTION}

The specifications of the $3^{\text {rd }}$ generation partnership project (3GPP) require data rates of at least $1 \mathrm{Gbit} / \mathrm{s}$ in the downlink of the future $5 \mathrm{G}$ indoor wireless networks [1]. One solution to achieve such throughput is to use millimeter waves (mmwaves) due to the large spectrum available in that range $(30$ $\mathrm{GHz}-300 \mathrm{GHz}$ ) [2]. Moreover, the high propagation losses in the mm-waves range [3] can be advantageous in indoor environments because it reduces interference and allows for frequency reuse. Hence, $\mathrm{mm}$-waves are considered as a strong candidate for $5 \mathrm{G}$.

The mixer plays a key role in the mm-wave communication system. It is essential for the signal up-conversion and downconversion at the transmitter and the receiver, respectively. There is a plethora of mixers in the mm-wave range, including: Schottky diodes mixers [4], superconductor-insulatorsuperconductor (SIS) mixers [5], and low temperature grown GaAs (LTG-GaAs) photomixers [6]. In addition, an unitraveling carrier photodiode (UTC-PD) was recently demonstrated as an optically pumped mixer (OPM) for mmwaves [7].

The UTC-PD OPM could offer interesting system opportunities compared to other types of mixers. For example, the UTC-PD has a wide frequency range of operation [7], and it can be monolithically integrated with laser and modulators on InP substrates [8], paving the way for a single chip photonic mm-wave transceiver. Moreover, the UTC-PD works at room temperature as opposed to SIS mixers that require cooling to

This project has received funding from the European Union's Horizon 2020 research and innovation programme under the Marie Sklodowska-Curie grant agreement No 642355.

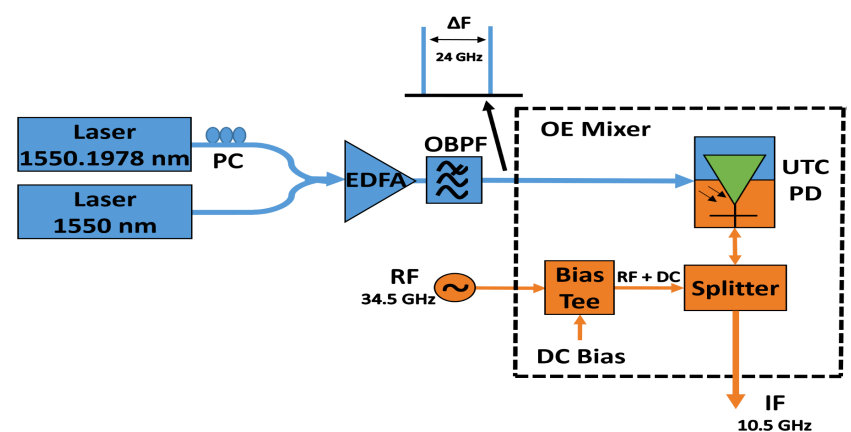

Fig. 1. Block diagram of OPM experiment using an UTC-PD.

very low temperatures. Further, the UTC-PD works at $1550 \mathrm{~nm}$ making it more attractive than LTG-GaAs photomixers, which work at $850 \mathrm{~nm}$ or shorter wavelength, since components and technologies in optical communications are easily available. Finally, unlike Schottky diode mixers, the UTC-PD does not require a millimeter wave electronic local oscillator (LO) as the LO can be generated by the UTC-PD itself using optical heterodyning [7].

It has already been shown that an UTC-PD can be used as an OPM at frequencies up to $600 \mathrm{GHz}$ [9], but these demonstrations were done within a $\mathrm{kHz}$ bandwidth $[7,10]$. In this paper, we demonstrate, for the first time, an OPM based on UTC-PD to down-convert a wireless RF signal modulated with 1 Gbaud quadrature phase shift keying (QPSK) data occupying a bandwidth of $1.35 \mathrm{GHz}$ and centered at $33.5 \mathrm{GHz}$. The signal was transmitted over a wireless distance of $1.4 \mathrm{~m}$, and the bit error rate (BER) of the received signal was estimated at $10^{-8}$.

Our experimental results demonstrate the potential of using the UTC-PD as a transceiver on a single chip like the one presented in [11]. This work could find interesting applications in future $5 \mathrm{G}$ or beyond networks since the $33 \mathrm{GHz}$ band is a candidate for $5 \mathrm{G}$ in a number of countries [12].

\section{UTC-PD MIXER CHARACTERIZATION}

In this work, an UTC-PD with epitaxy structure similar to the one presented in [13] was used as an OPM, by which an incoming radio frequency (RF) signal at $34.5 \mathrm{GHz}$ is mixed with an optically generated heterodyne signal $(\Delta \mathrm{F})$ at $24 \mathrm{GHz}$ to produce a new signal at an intermediate frequency (IF) at 10.5 GHz, as illustrated in Fig. 1. 


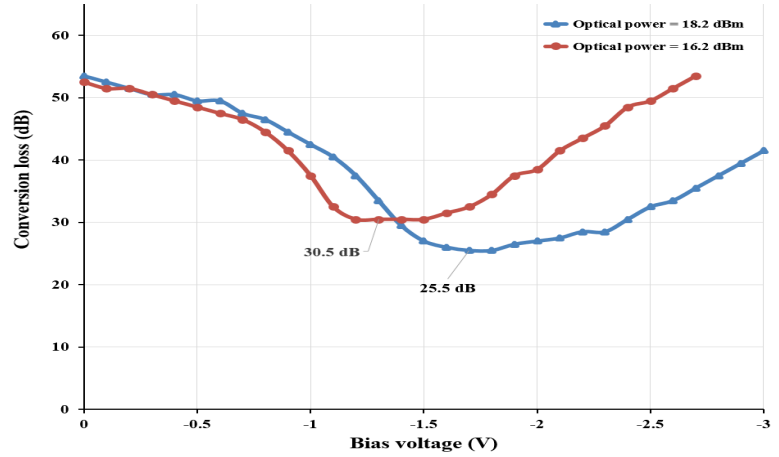

Fig. 2. UTC-PD's conversion loss vs. the voltage bias.

The Bias Tee, shown in Fig. 1, is used to couple the UTCPD bias to the RF signal, while the splitter is used to allow for the simultaneous supply of RF signal to the UTC-PD and the extraction of the generated IF signal.

The heterodyne signal $(\Delta \mathrm{F})$ was generated by injecting two optical tones into the UTC-PD mixer. The optical tones were generated by two free running external cavity lasers spaced by $0.1978 \mathrm{~nm}(24 \mathrm{GHz})$ and combined using a $3 \mathrm{~dB}$ optical coupler. Then, the optical signal was amplified by an Erbiumdoped fiber amplifier (EDFA) and filtered with a $1 \mathrm{~nm}$ optical bandpass filter (OBPF) to reduce the amplified spontaneous emission (ASE) noise. Finally, the optical signal was injected into the UTC-PD using a lensed fiber.

The mixing efficiency of the UTC-PD mixer depends on the level of the injected optical power into the UTC-PD and the applied bias voltage. Fig. 2 plots the conversion loss versus the bias voltage for different levels of injected optical power. Here, conversion loss is defined as the ratio of the power of the incoming unmodulated RF signal to the UTC-PD (measured at input of the coplanar probe that is connected to the waveguides of the UTC-PD), to the generated IF signal power. As it can be seen in the figure, when the voltage bias is increased, the conversion loss starts to decrease, up to the point where it reaches its minimum, after which it starts to increase again. Possible explanations of such behavior include: the modulation of the bias voltage by the heterodyne signal [10], and the modulation of the capacitance in the depletion layer [14]. The minimum conversion loss at $18.2 \mathrm{dBm}$ optical power was 25.5 $\mathrm{dB}$ obtained at $-1.7 \mathrm{~V}$, while it was $30.5 \mathrm{~dB}$ at $-1.3 \mathrm{~V}$ and 16.2 $\mathrm{dBm}$ optical power.

\section{WIRELESS TRANSMISSION EXPERIMENT}

Fig. 3 illustrates the block diagram of our wireless transmission experiment. An arbitrary waveform generator (AWG) was used to generate a 1 Gbaud QPSK signal centered at $1 \mathrm{GHz}$, and shaped with a root raised cosine (RRC) filter with a roll-off factor of 0.35 , resulting in a signal bandwidth of $1.35 \mathrm{GHz}$.

The QPSK signal was then up-converted using a doublebalanced electronic mixer that is driven by a $34.5 \mathrm{GHz}$ local oscillator (LO) resulting in two sidebands at $33.5 \mathrm{GHz}$ and 35.5 GHz. These two sidebands are identical and carry the same information. Therefore, detecting only one sideband is enough to recover the transmitted data.

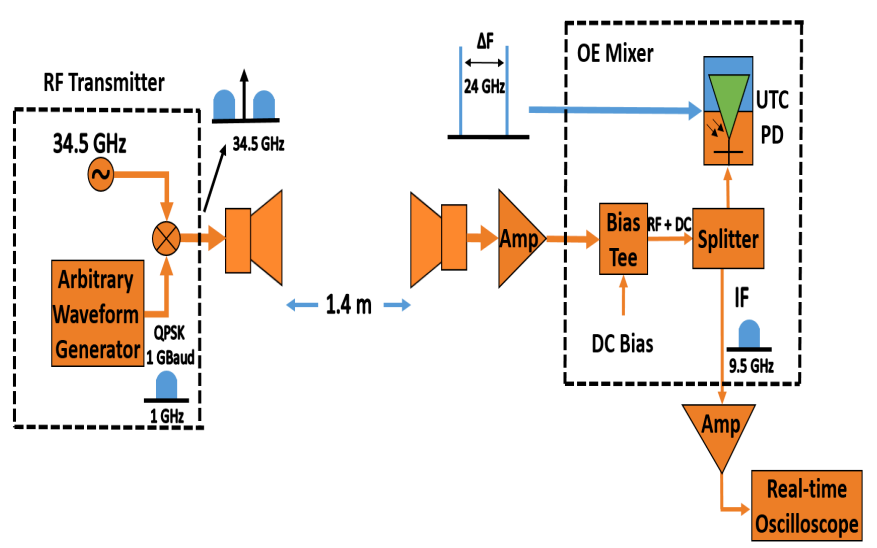

Fig. 3. Block diagram of the wireless transmission experiment.

The signal was transmitted using a $20 \mathrm{dBi}$ horn antenna over a wireless distance of $1.4 \mathrm{~m}$ before reaching a $20 \mathrm{dBi}$ horn antenna at the receiver. The received signal was amplified by a $40 \mathrm{GHz}$ RF amplifier $(\mathrm{G}=35 \mathrm{~dB}, \mathrm{NF}=4 \mathrm{~dB})$ and passed, through a bias tee and a splitter, onto a coplanar probe that is connected to the coplanar waveguides of the UTC-PD. The UTC-PD bias was set to $-1.7 \mathrm{~V}$, which gives the lowest conversion loss at $18.2 \mathrm{dBm}$ optical power (measured at the output of the OBPF).

The UTC-PD mixed the received RF carrier signal with the optical heterodyne, and down-converted the RF signal to an intermediate frequency (IF) of $10.5 \mathrm{GHz}$, resulting in the two sidebands centered at $9.5 \mathrm{GHz}$ and $11.5 \mathrm{GHz}$. The downconverted signal was then amplified $(\mathrm{G}=35 \mathrm{~dB}, \mathrm{NF}=4 \mathrm{~dB})$, then, captured by the real time oscilloscope, which has a bandwidth of $36 \mathrm{GHz}$ and a sampling rate of 80 Gsamples/second. The length of the captured time window was $5 \mu \mathrm{s}$, which corresponds to 10,000 bits. The captured signal was then bandpass filtered so that only the $9.5 \mathrm{GHz}$ sideband was passed for further digital signal processing. The filtered signal was digitally down-converted to the baseband, then, digitally filtered with an RRC filter with a roll-off factor of 0.35 . Fig. 4 shows the electrical spectrum of the signal after down-conversion to the baseband.

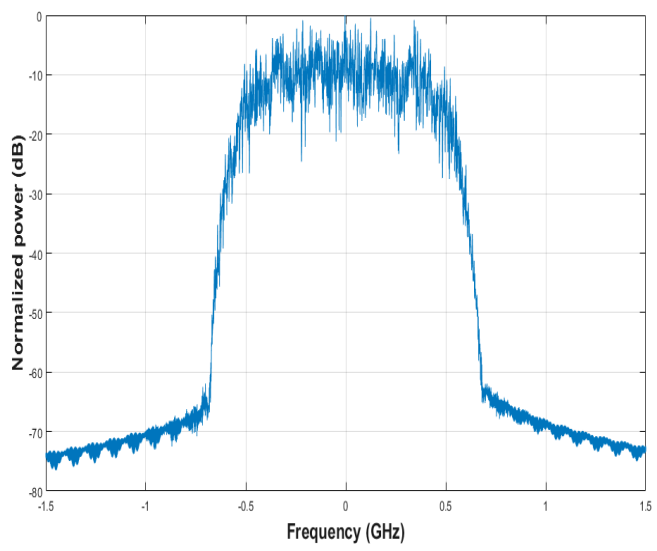

Fig. 4. Electrical spectrum of the received QPSK signal after down-conversion to the baseband. 


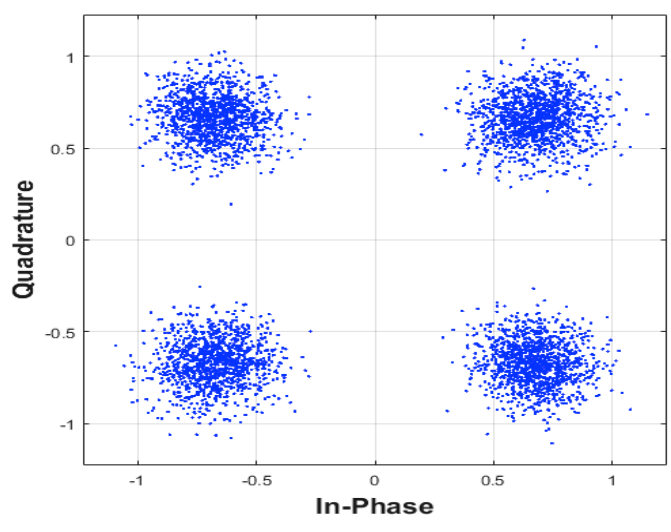

Fig. 5. Constellation diagram of the received QPSK signal after downconversion to the baseband.

Then, the constant modulus algorithm (CMA) was used for channel equalization. Also, we implemented carrier recovery and phase estimation algorithms to compensate for the frequency offset and phase drifting produced by the two free running laser since they are not locked to each other [15].

We were able to successfully recover the transmitted signal and reconstruct its constellation diagram as shown in Fig. 5. The measured error vector magnitude (EVM) of the received signal was $18 \%$. This corresponds to a BER of $10^{-8}$ [16], which would be sufficient for a transmission link of $2.5 \mathrm{~m}$ with forward error correction (FEC).

\section{CONCLUSION}

We have successfully demonstrated using the UTC-PD as a $2 \mathrm{Gbit} / \mathrm{s}$ wireless receiver. The UTC-PD down-converted a 1 Gbaud QPSK signal, after $1.4 \mathrm{~m}$ of wireless transmission. The BER of the received signal was estimated at $10^{-8}$.

This proof of concept was done at a carrier frequency of $34.5 \mathrm{GHz}$, where wireless propagation losses are low enough to easily assess the performance of the transmission system. This work can be easily extended to higher frequencies as the UTC$\mathrm{PD}$ offers frequency range of operation reaching hundreds of $\mathrm{GHz}$.

In this experiment, we used two free running lasers to generate the heterodyne signal on the UTC-PD. This provides high tuneability of the operating frequency simply by adjusting the wavelength spacing between the two lasers. Also, it allows for higher frequencies of operation without the need for an electronic local oscillator, which is the case in conventional electronic mixers.

Finally, the results of our experiment along with other experiments which previously demonstrated the UTC-PD as an mm-wave transmitter [17] show that there is a potential to use the UTC-PD as a transceiver on a chip like the one presented in [11].

\section{REFERENCES}

[1] T. Norp, "5G Service Requirements", 3gpp.org., 2017, Web. 24 May 2017

[2] Z. Pi and F. Khan, "An introduction to millimeter-wave mobile broadband systems," IEEE Communications Magazine, vol. 49, pp. 101-107, 2011.

[3] A. J. Seeds, H. Shams, M. J. Fice, and C. C. Renaud, "TeraHertz photonics for wireless communications," Journal of Lightwave Technology, vol. 33, pp. 579-587, 2015.

[4] S. Khanal, T. Kiuru, M. Hoefle, J. Montero, O. Cojocari, J. Mallat, P. Piironen, and A. V. Räisänen, "Characterisation of low-barrier Schottky diodes for millimeter wave mixer applications," in 2016 Global Symposium on Millimeter Waves, GSMM 2016 and ESA Workshop on Millimetre-Wave Technology and Applications, pp. 1-4, 2016.

[5] A. Navarrini, A. L. Fontana, D. Maier, P. Serres, and D. Billon-Pierron, "Superconductor-insulator-superconductor mixers for the $2 \mathrm{~mm}$ band (129-174 GHz)," Journal of Infrared, Millimeter, and Terahertz Waves, vol. 35, pp. 536-562, 2014.

[6] G. Seniutinas, G. Gervinskas, E. Constable, A. Krotkus, G. Molis, G. Valušis, R. A. Lewis, and S. Juodkazis, "THz photomixer with a 40nmwide nanoelectrode gap on low-temperature grown GaAs," Proc. SPIE, vol. 8923, pp. 892322-1 892322-9, 2013.

[7] E. Rouvalis, M. J. Fice, C. C. Renaud, and A. J. Seeds, "Millimeterwave optoelectronic mixers based on uni-traveling carrier photodiodes," IEEE Transactions on Microwave Theory and Techniques, vol. 60, pp. 686-691, 2012.

[8] H. Ito, S. Kodama, Y. Muramoto, T. Furuta, T. Nagatsuma, and T. Ishibashi, "High-speed and high-output InP-InGaAs unitraveling-carrier photodiodes," IEEE Journal on Selected Topics in Quantum Electronics, vol. 10, pp. 709-727, 2004.

[9] C. C. Renaud, M. J. Fice, L. Ponnampalam, M. Natrella, C. Graham, and A. J. Seeds, "Uni-travelling carrier photodetectors as $\mathrm{THz}$ detectors and emitters," Proc. SPIE, vol. 9370, pp. 93700B 1-93700B 9, 2015.

[10] E. Rouvalis, M. J. Fice, C. C. Renaud, and A. J. Seeds, "Optoelectronic detection of millimetre-wave signals with travelling-wave uni-travelling carrier photodiodes," Opt. Express, vol. 19, pp. 2079-2084, 2011.

[11] F. V. Dijk, G. Kervella, M. Lamponi, M. Chtioui, F. Lelarge, E. Vinet, Y. Robert, M. J. Fice, C. C. Renaud, A. Jimenez, and G. Carpintero, "Integrated InP heterodyne millimeter wave transmitter," IEEE Photonics Technology Letters, vol. 26, pp. 965-968, 2014.

[12] 4G Americas, "5G spectrum recommendations", 2015.

[13] M. Natrella, E. Rouvalis, C.-P. Liu, H. Liu, C. C. Renaud, and A. J. Seeds, "InGaAsP-based uni-travelling carrier photodiode structure grown by solid source molecular beam epitaxy," Opt. Express, vol. 20, pp. 19279-19288, 2012.

[14] H. Fushimi, T. Furuta, T. Ishibashi, and H. Ito, "Photoresponse Nonlinearity of a Uni-Traveling-Carrier Photodiode and Its Application to Optoelectronic Millimeter-Wave Mixing in $60 \mathrm{GHz}$ Band," Japanese Journal of Applied Physics, vol. 43, pp. L 966-L 968, 2004.

[15] H. Shams, M. J. Fice, K. Balakier, C. C. Renaud, F. van Dijk, and A. J. Seeds, "Photonic generation for multichannel $\mathrm{THz}$ wireless communication," Opt. Express, vol. 22, pp. 23465-23472, 2014.

[16] Fatadin, "Estimation of BER from Error Vector Magnitude for Optical Coherent Systems," Photonics, vol. 3, pp. 21, 2016.

[17] F. V. Dijk, G. Kervella, M. Lamponi, M. Chtioui, F. Lelarge, C. Renaud, G. Carpintero, and M. Alouini, "Wireless data transmission and frequency stabilization with a millimeter-wave photonic integrated circuit," in 2015 International Topical Meeting on Microwave Photonics (MWP), 2015. 